Received: 13.08 .2019

Revised: 18.08 .2019

Accepted: 30.08 .2019

DOI: $10.17804 / 2410-9908.2019 .4 .044-055$

\title{
THE EFFECT OF TANGENTIAL BOUNDARY STRESSES ON THE CONVECTIVE UNIDIRECTIONAL FLOW OF A VISCOUS FLUID LAYER UNDER THE LOWER BOUNDARY HEATING CONDITION
}

\author{
V. V. Privalova ${ }^{\text {a)**}}$ and E. Yu. Prosviryakov ${ }^{\text {b) }}$ \\ Institute of Engineering Science, Ural Branch of the Russian Academy of Sciences \\ 34 Komsomolskaya St., Ekaterinburg, 620049, Russian Federation \\ a) iD https://orcid.org/0000-0002-8648-0900 valentprival@gmail.com; \\ b) iD https://orcid.org/0000-0002-2349-7801 evgen_pros@mail.ru \\ *Corresponding author. E-mail: valentprival@gmail.com \\ Address for correspondence: 34 Komsomolskaya St., Ekaterinburg, 620049, Russian Federation \\ Tel.: +7 (343) 37535 76; fax: 3745330
}

An exact solution describing a convective unidirectional flow of a viscous incompressible fluid is obtained. The motion takes place in an infinite layer of some finite thickness. The fields of velocity, temperature, and pressure are time-independent. The resulting exact solution is a polynomial of various orders along the vertical (transverse) coordinate. A boundary value problem is formulated for the exact solution constructed. On the absolutely solid lower boundary of the fluid layer, the Navier slip condition and heating are specified. On the upper (free) surface, nonzero tangential stresses and pressure along the longitudinal coordinate are set. The temperature at the upper boundary is assumed to be zero. The obtained particular exact solution of the boundary value problem for the velocity function is analyzed. It is shown that, in the fluid layer under consideration, the velocity can have up to two stagnation points, which indicates the occurrence of countercurrent zones. The nonzero component of the vorticity vector and the tangential stress are analyzed. It is shown that the vorticity vector can change its sign in the fluid layer up to two times and that the tangential stress can change from tensile to compressive as many times.

Keywords: exact solution, counterflow, stagnation point, Navier slip condition, vorticity vector.

\section{References}

1. Roache P. J. Computational fluid dynamics, Hermosa Publs, Albuquerque, 1976, 446 p.

2. Fletcher C.A.J. Computational Techniques for Fluid Dynamics, Springer-Verlag, Berlin, Heidelberg, New York, London, Paris, Tokyo, 1988. DOI: 10.1007/978-3-642-97035-1.

3. Samarskii A.A. The Theory of Difference Schemes, Marcel Dekker Inc., New York, 2001, 786 p. DOI: $10.1201 / 9780203908518$.

4. $\quad$ Aristov S.N., Knyazev D.V., Polyanin A.D. Exact solutions of the Navier-Stokes equations with the linear dependence of velocity components on two space variables. Theor. Found. Chem. Eng., 2009, vol. 43, no. 5, pp. 642-662. DOI: 10.1134/S0040579509050066.

5. Lin C.C. Note on a class of exact solutions in magneto-hydrodynamics. Arch. Rational Mech. Anal., 1957, vol. 1, pp. 391-395. DOI: 10.1007/BF00298016.

6. Aristov S.N. and Shvarts K.G. Vikhrevye techeniya advektivnoy prirody vo vrashchayushchemsya sloe zhidkosti [Advective Eddy Flows in a Rotating Liquid Layer]. Perm, Permskiy Un-t. Publ., 2006. (In Russian).

7. Aristov S.N., Prosviryakov E.Yu. On laminar flows of planar free convection. Russian Journal of Nonlinear Dynamics, 2013, vol. 9, no. 4, pp. 651-657. DOI: 10.20537/nd1304004. 
8. Aristov S.N., Prosviryakov E.Yu. A new class of exact solutions for three-dimensional thermal diffusion equations. Theor. Found. Chem. Eng., 2016, vol. 50, no. 3, pp. 286-293. DOI: $10.1134 / \mathrm{S} 0040579516030027$.

9. Poiseuille J. Recherches expŭrimenteles sur le mouvement des liquides dans les tubes de trus petits diamutres. Comptes rendus hebdomadaires des süances de l'Acadmemie des Sciences, 1840, vol. 11, pp. 961-967, pp. 1041-1048.

10. Poiseuille J. Recherches expŭrimentales sur le mouvement des liquides dans les tubes de trus petits diamutres (suite). Comptes rendus hebdomadaires des süances de l'Acadmemie des Sciences, 1841, vol. 12, pp. 112-115.

11. Aristov S.N., Knyazev D.V. New exact solution of the problem of rotationally symmetric Couette-Poiseuille flow. J. Appl. Mech. Tech. Phys., 2007, vol. 48, no. 5, pp. 680-685. DOI: $10.1007 / \mathrm{s} 10808-007-0087-7$.

12. Regirer S.A. On the movement of fluid in a tube with a deforming wall. Izv. AN SSSR. Mechanika Zhidkosti i Gaza, 1968, no. 4, pp. 202-204.

13. Regirer, S.A. Quasi-one-dimensional theory of peristaltic flows. Fluid Dyn., 1984, vol. 19, no. 5, pp. 747-754. DOI: 10.1007/BF01093542.

14. Takagi D., Balmforth N.J. Peristaltic pumping of rigid objects in an elastic tube. J. Fluid Mech., 2011, vol. 672, pp. 219-244. DOI: 10.1017/S0022112010005926.

15. Yin F.C.P., Fung Y.C. Peristaltic waves in circular cylindrical tubes. Trans. ASME. J. Appl. Mech., 1969, vol. 36, pp. 579-587.

16. Pedley T. The Fluid Mechanics of Large Blood Vessels (Cambridge Monographs on Mechanics), Cambridge University Press, Cambridge, 1980. DOI: 10.1017/CBO9780511896996.

17. Medvedev A.E. Three-dimensional motion of a viscous incompressible fluid in a narrow tube. J. Appl. Mech. Tech. Phys., 2009, vol. 50, no. 4, pp. 566-569. DOI: 10.1007/s10808-009-0076-0.

18. Medvedev A.E. Unsteady motion of a viscous incompressible fluid in a tube with a deformable wall. J. Appl. Mech. Tech. Phys., 2013, vol. 54, no. 4, pp. 552-560. DOI: 10.1134/S0021894413040056.

19. Tverier V.M., Gladysheva O.S. A biomechanical model of the mammary gland. Master's Journal, 2013, no. 2, pp. 240-252. (In Russian).

20. Gavrilenko S.L., Vasin R.A., and Shilko S.V. A method for determining flow and rheological constants of viscoplastic biomaterials. Part 1. Russian Journal of Biomechanics, 2002, vol. 6, no. 3, pp. 92-99. (In Russian).

21. Shil'ko S.V., Gavrilenko S.L., Khizhenok V.F., Stakan I.N., and Salivonchik S.P. A method for defining flow and rheological constants of viscoplastic biomaterials. Part 2. Russian Journal of Biomechanics, 2003, vol. 7, no. 2, pp. 79-84. (In Russian).

22. Goldstein S. Modern Developments in Fluid Mechanics, Oxford Univ. Press, Oxford, 1938.

23. Neto C., Evans D., Bonaccurso E. Boundary slip in Newtonian liquids: a review of experimental studies. Reports on Progress in Physics, 2005, vol. 39, pp. 2859-2897. DOI: 10.1088/00344885/68/12/R05.

24. Yankov V.I., Boyarchenko V.I., Pervadchuk V.P., Glot I.O., Shakirov N.V. Pererabotka voloknoobrazuyushchikh polimerov. Osnovy reologii polimerov $i$ techeniye polimerov $v$ kanalakh [Processing of fiber-forming polymers. Bases of polymer rheology and polymer flow in channels]. Moscow-Izhevsk, RKhD Publ., 2008, 264 p. (In Russian).

25. Vinogradova O.I., Belyaev A.V. Wetting, roughness and flow boundary conditions. J. Phys. Condens. Matter, 2011, vol. 23, pp. 184104 (1-15). DOI: 10.1088/0953-8984/23/18/184104.

26. Belyaev A.V., Vinogradova O.I. Effective slip in pressure-driven flow past super-hydrophobic stripes. J. Fluid Mech., 2010, vol. 652, pp. 489-499. DOI: 10.1017/S0022112010000741.

27. Borzenko E.I., Diakova O.A., Shrager G.R. Studying the slip phenomenon for a viscous fluid flow in a curved channel. Vestn. Tomsk. Gos. Univ. Mat. Mekh., 2014, no. 2 (28), pp. 35-44. (In Russian). 
28. Itoh S., Tanaka N., Tani A. The initial value problem for the Navier-Stokes equations with general slip boundary conditions in Holder spaces. J. Math, Fluid Mech., 2003, vol. 5, pp. 275-301. DOI: $10.1007 / \mathrm{s} 00021-003-0074-6$.

29. Beirao da Veiga H. Regularity for Stokes and generalized Stokes systems under nonhomogeneous slip-type boundary conditions. Advances in Differential Equations, 2004, vol. 9, no. 9-10, pp. 1079-1114.

30. Fugita H. Non-stationary Stokes flows under leak boundary conditions of friction type. J. Comput. Math., 2001, vol. 19, no. 1, pp. 1-8.

31. Gershuni G.Z., Zhukhovitskii E.M. Convective Stability of Incompressible Fluids. Israel Program for Scientific Translations. Jerusalem, Keter Publishing House, 1976, 330 p.

32. Burmasheva N.V., Prosviryakov E.Yu. A large-scale layered stationary convection of an incompressible viscous fluid under the action of shear stresses at the upper boundary. Velocity field investigation. Vestn. Samar. Vestn. Samar. Gos. Tekhn. Univ., Ser. Fiz.-Mat. Nauki, 2017, vol. 21, no. 1, pp. 180-196. DOI: 10.14498/vsgtu1527. (In Russian).

33. Privalova V.V., Prosviryakov E.Yu., Couette-Hiemenz Exact Solutions for the Steady Creeping Convective Flow of a Viscous Incompressible Fluid, with Allowance Made for Heat Recovery. J. Samara State Tech. Univ., Ser. Phys. Math. Sci., 2018, vol. 22, no. 3, pp. 1-17. DOI: $10.14498 /$ vsgtu1638.

34. Privalova V.V., Prosviryakov E.Yu. Exact solutions for the convective creep CouetteHiemenz flow with the linear temperature distribution on the upper border. Diagnostics, Resource and Mechanics of materials and structures, 2018, iss. 2, pp. 92-109. DOI: 10.17804/24109908.2018.2.092-109.

35. Aristov S.N., Prosviryakov E.Yu. Unsteady layered vortical fluid flows. Fluid Dynamics, 2016, vol. 51, no. 2, pp. 148-154. DOI: 10.1134/S0040579516030027.

36. Navier C.L.M.H. M'emoire sur les Lois du Mouvement des Fluides. M'em. Acad. Sci. Inst. de France, 1822, vol. 2, no. 6, pp. 389-440.

37. Navier C.L.M.H. Sur les lois du mouvement des fluids. M'em. Acad. R. Sci. Inst., 1827, fr. 6, pp. 389-440. 
Подана в журнал: 13.08 .2019

УДК 532.51

DOI: $10.17804 / 2410-9908.2019 .4 .044-055$

\title{
ВЛИЯНИЕ КАСАТЕЛЬНЫХ ГРАНИЧНЫХ НАПРЯЖЕНИЙ НА КОНВЕКТИВНОЕ ОДНОНАПРАВЛЕННОЕ ТЕЧЕНИЕ СЛОЯ ВЯЗКОЙ ЖИДКОСТИ ПРИ УСЛОВИИ НАГРЕВА НИЖНЕЙ ГРАНИЦЫ
}

\author{
В. В. Привалова ${ }^{\text {a)* }}$ Е. Ю. Просвиряков ${ }^{\text {) }}$ \\ Институт машиноведения Уральского отделения Российской академии наук, \\ д. 34, ул. Комсомольская, г. Екатеринбург, Российская Федерация \\ a) iD https://orcid.org/0000-0002-8648-0900 valentprival@ gmail.com; \\ б) (iD https://orcid.org/0000-0002-2349-7801 《evgen_pros@mail.ru \\ *Ответственный автор. Электронная почта: valentprival@gmail.com \\ Адрес для переписки: ул. Комсомольская, 34, г. Екатеринбург, Российская Федерация \\ Тел.: +7 (343) 375-35-76; факс: 374-53-30
}

Получено точное решение, описывающее конвективное однонаправленное течение вязкой несжимаемой жидкости. Движение происходит в бесконечном слое некоторой конечной толщины. Поля скорости, температуры и давления не зависят от времени. Полученное точное решение представляет собой полиномы различных порядков по вертикальной (поперечной) координате. Для построенного точного решения сформулирована краевая задача. На нижней, абсолютно твердой границе слоя жидкости задается условие проскальзывания Навье и нагрев. На верхней (свободной) поверхности задаются ненулевые касательные напряжения и переменное по продольной координате давление. Температура на верхней границе полагается равной нулю. Проанализировано полученное частное точное решение краевой задачи для функции скорости. В рассматриваемом слое жидкости скорость может иметь до двух застойных точек, что говорит о возникновении зон противотечений. Проанализированы ненулевая компонента вектора завихренности и касательное напряжение. Показано, что вектор завихренности может менять свой знак в слое жидкости до двух раз, а касательное напряжение столько же раз может меняться с растягивающего на сжимающее.

Ключевые слова: точное решение, противотечение, застойная точка, условие проскальзывания Навье, вектор завихренности.

\section{1. Введение}

При моделировании технологических процессов, включающих движение сплошных сред, возникает потребность в описании течения жидкостей и газов. В случае, когда исследуемые среды можно считать несжимаемыми, удобным инструментом описания движения является уравнение Навье-Стокса и его приближения. Эти уравнения весьма сложны для интегрирования по причине сильной нелинейности, что влечет за собой наиболее частое использование аппарата численных методов нахождения решений [1-3]. Тем актуальнее становится задача отыскания точных решений для описания движения вязких несжимаемых сред. Не стоит также забывать, что именно точные решения позволяют наиболее результативно оценивать области применимости гидродинамических моделей и являются необходимым инструментом для апробации и тестирования асимптотических, приближенных и численных методов.

В свое время для интегрирования уравнений Навье-Стокса были предложены классы точных решений [4]. Одним из наиболее часто используемых классов является класс решений Линя [5]. Изначально эта методика была предложена для решения задач магнитной гид- 
родинамики. В этом семействе решений гидродинамические поля описываются функциями, линейными по части горизонтальных координат. В дальнейшем класс Линя дал возможность строить классы решений также для конвективных [6, 7] и термодиффузионных [8] задач.

Отдельный интерес при изучении движения вязкой несжимаемой жидкости вызывают градиентные течения, или течения Пуазейля $[9,10]$ с различными модификациями [11-15]. Это связано с достаточно высоким практическим применением такой модели в науке, технике, медицине и биологии. В частности, продольным изменением давления в канале или между плоскостями сопровождаются такие процессы, как движение крови по сосудам [16-18], процесс функционирования молочной железы в период лактации [19], а также для идентификации моделей биотканей [20, 21].

При рассматрении классов решений, описывающих движение вязкой несжимаемой жидкости, отдельным важным моментом является выбор граничных условий для формулирования краевых задач, возникающих в каждой отдельной модели течения сред. Длительное время при задании поля скорости жидкости на границе соприкосновения с твердой стенкой было принято задавать условие прилипания (нулевых скоростей) [22]. В последние десятилетия эта тенденция стала стремительно меняться в связи с развитием технологий, основанных на более микроскопическом уровне изучения области соприкосновения жидкости и ограничивающей ее поверхности [23-26]. Таким образом, все чаще в практических задачах стали рассматриваться условие проскальзывания и, как частный идеализированный случай, условие идеального скольжения [23, 27-30].

В работе предложено точное решение, относящее к классу, линейно зависящих от части координат. Краевая задача описывает конвективное однонаправленное течение слоя вязкой несжимаемой жидкости и включает задание условия проскальзывания на поверхности контакта жидкости с твердой поверхностью, а также ненулевых касательных напряжений на свободной поверхности.

\section{2. Постановка задачи}

Рассматриваем конвективное однонаправленное течение бесконечного слоя вязкой несжимаемой жидкости. Система уравнений, описывающая такое движение в векторном виде, включает уравнение Навье-Стокса в приближении Буссинеска, уравнение теплопроводности и уравнение несжимаемости [31]:

$$
\begin{gathered}
\frac{d \mathbf{V}}{d t}+(\mathbf{V}, \nabla) \mathbf{V}=-\nabla P+\nu \Delta \mathbf{V}+\mathbf{g} \beta T ; \\
\mathbf{V} \cdot \nabla T=\chi \Delta T \\
\nabla \cdot \mathbf{V}=0 .
\end{gathered}
$$

Здесь $\mathbf{V}(t, x, y, z)=\left(V_{x}, 0,0\right)$ - вектор скорости и его проекции на оси декартовой системы координат; $P$ - отклонение давления от гидростатического, отнесенное к постоянной средней плотности жидкости $\rho ; \mathbf{g}$ - вектор ускорения свободного падения; $\beta$ - температурный коэффициент объемного расширения жидкости; $T$ - отклонение от средней температуры; $v, \chi$ - коэффициенты кинематической вязкости и температуропроводности жидкости соответственно. Оси декартовой системы координат расположены таким образом, что течение жидкости происходит в направлении оси $O x$. Ось $O z$ направлена перпендикулярно направлению движения от нижней твердой границе слоя к верхней свободной поверхности. 
Полагаем, что движение жидкости установившееся, т. е. поля скорости, температуры и давления не зависят от времени. Таким образом, система уравнений (1)-(3) в проекциях на оси декартовой системы координат с учетом принятых допущений принимает вид:

$$
\begin{array}{cc}
v \frac{\partial^{2} V_{x}}{\partial z^{2}}=\frac{\partial P}{\partial x} ; \quad \frac{\partial P}{\partial y}=0 ; & \frac{\partial P}{\partial z}=g \beta T ; \\
V_{x} \frac{\partial T}{\partial x}=\chi\left(\frac{\partial^{2} T}{\partial x^{2}}+\frac{\partial^{2} T}{\partial y^{2}}+\frac{\partial^{2} T}{\partial z^{2}}\right) ; & \frac{\partial V_{x}}{\partial x}=0 .
\end{array}
$$

Решение системы уравнений (4) ищем в следующем виде [32-35]:

$$
\begin{gathered}
V_{x}=U(z) ; \quad V_{y}=0 ; \quad V_{z}=0 ; \\
P=P_{0}(z)+x P_{1}(z) ; \quad T=T_{0}(z)+x T_{1}(z) .
\end{gathered}
$$

Подставляем класс точных решений (5) в систему уравнений (4). С учетом того, что все искомые функции $U, P_{0}, P_{1}, T_{0}, T_{1}$, в принятом виде решения (5) зависят только от вертикальной (поперечной) координаты $z$, будем обозначать все производные по этой переменной штрихом. Используем метод неопределенных коэффициентов и получаем следующую систему уравнений записанную в порядке дальнейшего интегрирования:

$$
T_{1}^{\prime \prime}=0, P_{1}^{\prime}=g \beta T_{1}, v U^{\prime \prime}=P_{1} ; \quad \chi T_{0}^{\prime \prime}=U T_{1}, P_{0}^{\prime}=g \beta T_{0} .
$$

Система (6) состоит из пяти дифференциальных уравнений для определения пяти неизвестных функций.

\section{3. Общее решение системы уравнений}

Общее решение системы уравнений (6) записываем в следующем виде:

$$
\begin{gathered}
T_{1}=C_{1} z+C_{2} ; P_{1}=\frac{g \beta}{2}\left(z C_{1}+2 C_{2}\right) z+C_{3} ; \\
U=\frac{g \beta z^{3}}{4 ! v}\left(z C_{1}+4 C_{2}\right)+\frac{z^{2}}{2 v} C_{3}+z C_{4}+C_{5} ; \\
T_{0}=\frac{g \beta z^{5}}{7 ! v \chi}\left(5 z^{2} C_{1}^{2}+35 z C_{1} C_{2}+42 C_{2}^{2}\right)+\frac{z^{4} C_{3}}{5 ! v \chi}\left(3 z C_{1}+5 C_{2}\right)+ \\
+\frac{2 z^{2}}{4 ! \chi}\left[z^{2} C_{1} C_{4}+6 C_{2} C_{5}+2 z\left(C_{2} C_{4}+C_{1} C_{5}\right)\right]+z C_{6}+C_{7} ; \\
P_{0}=\frac{g^{2} \beta^{2} z^{6}}{8 ! v \chi}\left(5 z^{2} C_{1}^{2}+40 z C_{1} C_{2}+56 C_{2}^{2}\right)+g \beta\left\{\frac{3 z^{5} C_{3}}{6 ! v \chi}\left(z C_{1}+2 C_{2}\right)+\right. \\
\left.+\frac{z^{3}}{5 ! \chi}\left[2 z^{2} C_{1} C_{4}+20 C_{2} C_{5}+5 z\left(C_{2} C_{4}+C_{1} C_{5}\right)\right]+\frac{1}{2} z^{2} C_{6}+z C_{7}\right\}+C_{8} .
\end{gathered}
$$

Здесь постоянные интегрирования $C_{i}(i=\overline{1,8})$ определяются заданием граничных условий. 


\section{4. Точное решение краевой задачи с условием проскальзывания Навье и нагревом на нижней границе}

Находим частное точное решение для системы уравнений (6). Для этого записываем граничные условия для рассматриваемого слоя жидкости. Полагаем нижнюю границу бесконечного горизонтального слоя жидкости, задаваемого уравнением $z=0$, абсолютно твердой и неподвижной. На нижней границе задан нагрев и условие проскальзывания Навье [23, 24, $27,36,37]$. На верхней (свободной) поверхности, определяемой уравнением $z=h$, заданы ненулевые касательные напряжения и переменное по продольной координате $x$ давление. Температура на верхней границе равна отсчетному нулевому значению. Таким образом, граничные условия можно записать в следующем виде:

$$
T(0)=A x ;\left.\alpha \frac{\partial V_{x}}{\partial z}\right|_{z=0}=V_{x}(0) ; T(h)=0 ; P(h)=S_{0}+S_{1} x ;\left.\frac{\partial V_{x}}{\partial z}\right|_{z=h}=\tau \text {. }
$$

С учетом структуры (5) выбранного обобщенного класса решений граничные условия (8) для решения краевой задачи можно переписать в следующем виде:

$$
\begin{array}{ccc}
T_{0}(0)=0 ; & T_{1}(0)=A ; & \left.\alpha \frac{\partial U}{\partial z}\right|_{z=0}=U(0) ; \\
T_{0}(h)=T_{1}(h)=0 ; & P_{0}(h)=S_{0} ; \quad P_{1}(h)=S_{1} ;\left.\quad \frac{\partial U}{\partial z}\right|_{z=h}=\tau .
\end{array}
$$

Здесь $\alpha$ - размерный коэффициент проскальзывания (длина проскальзывания).

С учетом граничных условий (9) постоянные интегрирования $C_{i}(i=\overline{1,8})$ для общего решения (7) вычисляются следующим образом:

$$
\begin{gathered}
C_{1}=-\frac{A}{h} ; \quad C_{2}=A ; \quad C_{3}=-\frac{1}{2} A g h \beta+S_{1} ; \\
C_{4}=\frac{1}{6 v}\left(A h^{2} g \beta-6 \tau-6 h S_{1}\right) ; \quad C_{5}=\frac{\alpha}{6 v}\left(A h^{2} g \beta-6 \tau-6 h S_{1}\right) \\
C_{6}-\frac{A^{2} h^{3} g \beta}{126 v \chi}(h+7 \alpha)+\frac{A h \alpha}{3 v \chi}\left(\tau+h S_{1}\right)+\frac{A h^{2}}{1260 v \chi}\left(105 \tau+84 h S_{1}\right) ; \quad C_{7}=0 \\
C_{8}=S_{0}+\frac{A^{2} h^{5} g^{2} \beta^{2}}{8064 v \chi}(11 h+56 \alpha)-\frac{A h^{3} g \beta}{24 v \chi}\left[\alpha\left(\tau+h S_{1}\right)+\frac{h}{10}\left(4 \tau+3 h S_{1}\right)\right]
\end{gathered}
$$

Подставляем полученные выражения постоянных интегрирования (10) в общее решение (7) и получаем частное точное решение краевой задачи (6), (9) в виде полиномов:

$$
\begin{gathered}
T_{1}=A\left(1-\frac{z}{h}\right) ; \quad P_{1}=S_{1}-\frac{A g \beta}{2 h}\left(z^{2}-2 h z+h^{2}\right) ; \\
U=\frac{S_{1}}{2 v}\left[z^{2}-2 h(z+\alpha)\right]-\frac{A g \beta}{24 h v}\left[z^{4}-4 h z^{3}+6 h^{2} z^{2}-4 h^{3}(z+\alpha)\right]-\frac{\tau}{v}(z+\alpha) ; \\
T_{0}=\frac{A z}{1008 h^{2} v \chi}\left(2 h^{2}-3 h z+z^{2}\right)\left\{A g \beta\left[z^{4}-4 h z^{3}+7 h^{2} z^{2}-2 h^{3}(3 z+14 \alpha)-4 h^{4}\right]+\right.
\end{gathered}
$$




$$
\begin{gathered}
\left.+168 h \alpha\left(\tau+h S_{1}\right)\right\}+\frac{A}{12 h v \chi}\left[z\left(h^{3}-2 h z^{2}+z^{3}\right) \tau+\frac{S_{1}}{10}\left(8 h^{4} z-20 h^{2} z^{3}+15 h z^{4}-3 z^{5}\right)\right] \\
P_{0}=S_{0}+\frac{A^{2} g^{2} \beta^{2}}{8064 h^{2} v \chi}\left(11 h^{8}-32 h^{6} z^{2}+56 h^{4} z^{4}-56 h^{3} z^{5}+28 h^{2} z^{6}-8 h z^{7}+z^{8}\right)+ \\
\quad+\frac{A \alpha g \beta}{144 h v \chi}(h-z)^{2}\left(h^{2}+2 h z-z^{2}\right)\left(A g h^{2} \beta-6 \tau-6 h S_{1}\right)- \\
-\frac{A g \beta}{240 h v \chi}(h-z)^{2}\left[2\left(2 h^{3}+4 h^{2} z+h z^{2}-2 z^{3}\right) \tau+\left(3 h^{4}+6 h^{3} z+h^{2} z^{2}-4 h z^{3}+z^{4}\right) S_{1}\right] .
\end{gathered}
$$

Полученные полиномы имеют степени от первой (функция $T_{1}$ - частная производная от температуры по продольной координате $x$ ) до восьмой (функция $P_{0}$ - однородная составляющая давления).

\section{5. Анализ поля скоростей}

Проведем анализ полученной компоненты скорости $U$. Исследуем возможность существования нулевых (застойных) точек функции скорости $U$ и их количество в слое жидкости при изменении вертикальной переменной $z \in(0 ; h)$.

Скорость определяется взаимодействием трех потоков: потока, вызванного перепадом давления (пуазейлевского потока); потока, вызванного нагревом/охлаждением и действием силы тяготения (термогравитационного потока); потока, возникающего под действием касательного напряжения на верхней границе слоя жидкости (ветровой поток).

Представим функцию скорости $U$ в следующем виде:

$$
U=\frac{S_{1}}{2 v} f_{1}-\frac{A g \beta}{24 h v} f_{2}-\frac{\tau}{v} f_{3}
$$

Здесь исследуемые функции $f_{1}, f_{2}$ и $f_{3}$, зависимые от переменной выражают следующие полиномы:

$$
\begin{gathered}
f_{1}=z^{2}-2 h(z+\alpha) \\
f_{2}=z^{4}-4 h z^{3}+6 h^{2} z^{2}-4 h^{3}(z+\alpha) \\
f_{3}=z+\alpha
\end{gathered}
$$

Для нахождения застойных точек скорости $U$ в рассматриваемом слое жидкости проанализируем сначала возможность существования нулевых точек у составляющих $f_{1}, f_{2}$ и $f_{3}$. Функция $f_{1}$ обращается в нуль при $z=h \pm \sqrt{h^{2}+2 h \alpha}$. Для того чтобы найденные нулевые точки принадлежали рассматриваемому слою, необходимо выполнение следующего двойного неравенства:

$$
0<h \pm h \sqrt{1+\frac{2 \alpha}{h}}<h .
$$


Полученное неравенство (13) не выполняется ни при каких положительных $\alpha$ и $h$. Следовательно, функция $f_{1}$ не имеет корней на интервале $(0 ; h)$.

Аналогично исследуем возможность существования корней у функции $f_{2}$. Функция $f_{2}$ обращается в нуль при $z=h \pm \sqrt[4]{h^{4}+4 h^{3} \alpha}$. Найденные значения переменной $z$ принадлежат интервалу $(0 ; h)$ при выполнении следующего двойного неравенства:

$$
0<h \pm h \sqrt[4]{1+\frac{4 \alpha}{h}}<h
$$

Полученное неравенство (14) также не выполняется ни при каких положительных $\alpha$ и $h$. Следовательно, функция $f_{2}$ не имеет корней на интервале $(0 ; h)$.

Функция $f_{3}$ на интервале $(0 ; h)$ всюду положительна и не принимает нулевых значений.

Исследуя совместное влияние трех потоков, вызванных перепадом давления, нагревом/охлаждением и действием касательного напряжения на функцию скорости получено, что функция скорости $U$ при определенном подборе параметров, заданных на границе, может иметь до двух застойных точек на рассматриваемом интервале $(0 ; h)$. Профиль функции скорости $U$ при двух застойных точках представлен на рис. 1.

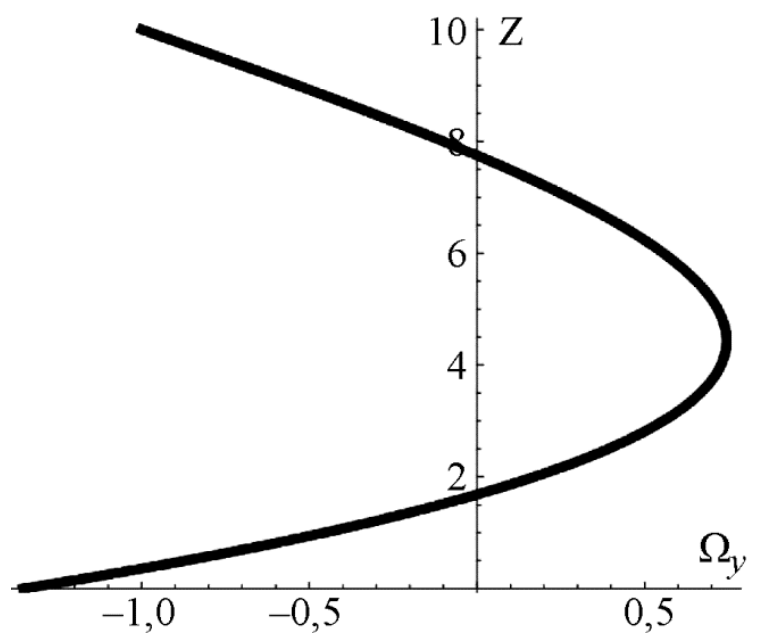

Рис. 1. Профиль функции скорости $U$ при следующих значениях параметров:

$$
\begin{gathered}
v=10^{-6} \mathrm{M}^{2} / \mathrm{c} ; g=9,8 \mathrm{M} / \mathrm{c}^{2} ; \beta=1,82 \cdot 10^{-4} 1 / \mathrm{K} ; h=10 \mathrm{M} ; A=-1,7 \cdot 10^{-4} ; \\
\tau=10^{-6} \mathrm{M} / \mathrm{c}^{2} ; S_{1}=-4,7 \cdot 10^{-7} \mathrm{M} / \mathrm{c}^{2}
\end{gathered}
$$

\section{5. Исследование касательных напряжений и завихренности}

Исследуем вектор завихренности $\Omega=\left(\Omega_{x}, \Omega_{y}, \Omega_{z}\right)$ и касательные напряжения полученного частного точного решения (11). В случае однонаправленного течения только одна компонента вектора завихренности отлична от нуля. Записываем выражение ненулевой компоненты $\Omega_{y}$ и соответствующее ей касательное напряжение, определяемые частной производной компоненты скорости: 


$$
\Omega_{y}=\frac{\partial V_{x}}{\partial z}=\tau_{x z}
$$

Таким образом, для полученного частного точного решения ненулевая компонента вектора завихренности $\Omega_{y}$ имеет вид:

$$
\Omega_{y}=\frac{1}{v}\left[\frac{A g \beta}{24 h}\left(4 h^{3}-12 h^{2} z+12 h z^{2}-4 z^{3}\right)-\tau+(z-h) S_{1}\right] .
$$

На рис. 2 представлен профиль компоненты завихренности $\Omega_{y}$ при тех же значениях параметров, при которых на рис. 1 изображен профиль скорости с двумя застойными точками на интервале $(0 ; h)$. Как видно, компонента завихренности $\Omega_{y}$ дважды в слое жидкости меняет знак на противоположный.

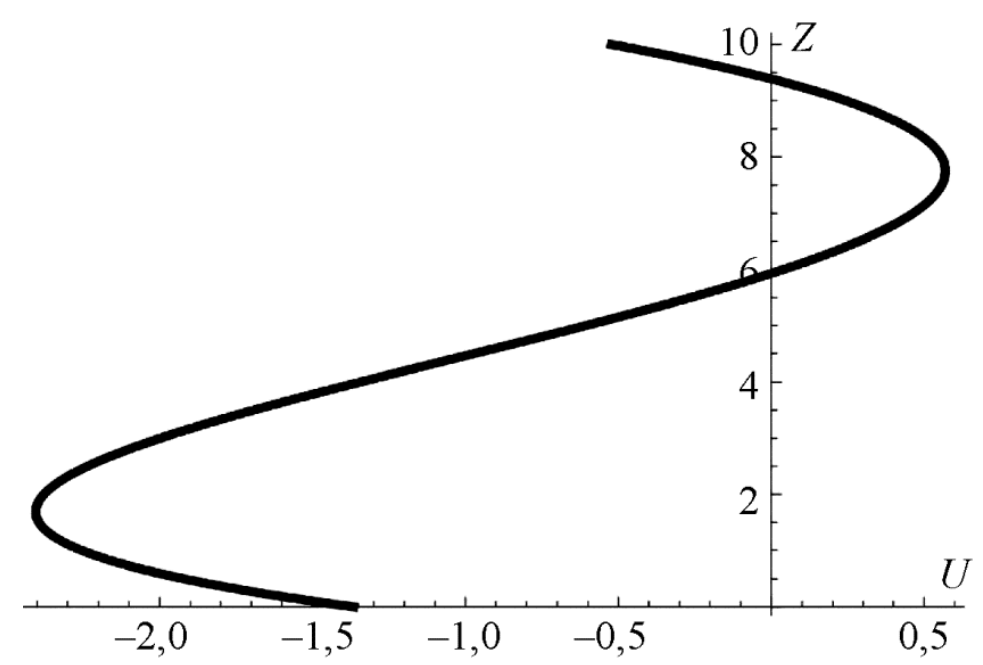

Рис. 2. Профиль компоненты завихренности $\Omega_{y}$ при следующих значениях параметров:

$$
\begin{gathered}
v=10^{-6} \mathrm{M}^{2} / \mathrm{c} ; g=9,8 \mathrm{M} / \mathrm{c}^{2} ; \beta=1,82 \cdot 10^{-4} 1 / \mathrm{K} ; h=10 \mathrm{M} ; A=-1,7 \cdot 10^{-4} ; \\
\tau=10^{-6} \mathrm{M} / \mathrm{c}^{2} ; S_{1}=-4,7 \cdot 10^{-7} \mathrm{M} / \mathrm{c}^{2}
\end{gathered}
$$

Учитывая зависимость (15) можно сделать заключение о том, что касательное напряжение $\tau_{x z}$ на интервале $(0 ; h)$ может меняться с растягивающего на сжимающее до двух раз.

\section{6. Заключение}

Получено точное решение для конвективного однонаправленного течения вязкой несжимаемой жидкости. В предложенном решении поле скорости рассматривалось как однородный по продольным координатам полином, зависящий только от вертикальной (поперечной) координаты. Поля температуры и давления представлены полиномами с неоднородной частью по горизонтальной (продольной) координате. Для предложенного вида точного решения решена краевая задача и проанализировано полученное поле скоростей. Показано, что в рассматриваемом слое жидкости скорость может иметь до двух застойных точек. В этих же точках вектор завихренности меняет знак на противоположный, а касательное напряжение трансформируется из растягивающего в сжимающее или наоборот. 


\section{Литература}

1. Роуч П. Вычислительная гидродинамика. - М. : Мир, 1980. - 616 с.

2. Флетчер К. Вычислительные методы в динамике жидкостей : в двух томах / пер. с англ. - М. : Мир, 1991.

3. Самарский А. А. Теория разностных схем. - М. : Наука, 1977. - 656 с.

4. Aristov S. N., Knyazev D. V., Polyanin A. D. Exact solutions of the Navier-Stokes equations with the linear dependence of velocity components on two space variables // Theor. Found. Chem. Eng. - 2009. - Vol. 43, no. 5. - P. 642-662. - DOI: 10.1134/S0040579509050066.

5. Lin C. C. Note on a class of exact solutions in magneto-hydrodynamics// Arch. Rational Mech. Anal. - 1958. - Vol. 1. - P. 391-395. - DOI: 10.1007/BF00298016.

6. Аристов С. Н., Шварц К. Г. Вихревые течения адвективной природы во вращающемся слое жидкости. - Пермь : Пермский ун-т, 2006. - 155 с.

7. Aristov S. N., Prosviryakov E. Yu. On laminar flows of planar free convection // Russian Journal of Nonlinear Dynamics. - 2013. - Vol. 9, no. 4. - P. 651-657. - DOI: 10.20537/nd1304004.

8. Aristov S. N., Prosviryakov E. Yu. A new class of exact solutions for three-dimensional thermal diffusion equations // Theor. Found. Chem. Eng. - 2016. - Vol. 50, no. 3. - P. 286-293. DOI: $10.1134 / \mathrm{S} 0040579516030027$.

9. Poiseuille J. Recherches expŭrimenteles sur le mouvement des liquides dans les tubes de trus petits diamutres // Comptes rendus hebdomadaires des sŭances de l'Acadmemie des Sciences. 1840. - Vol. 11. - P. 961-967. - P. 1041-1048.

10. Poiseuille J. Recherches expйrimentales sur le mouvement des liquides dans les tubes de trus petits diamutres (suite) // Comptes rendus hebdomadaires des sŭances de l'Acadmemie des Sciences. - 1841. - Vol. 12. - P. 112-115.

11. Aristov S. N., Knyazev D. V. New exact solution of the problem of rotationally symmetric Couette-Poiseuille flow // J. Appl. Mech. Tech. Phys. - 2007. - Vol. 48, no. 5. - P. 680-685. DOI: $10.1007 / \mathrm{s} 10808-007-0087-7$.

12. Регирер С. А. О движении жидкости в трубке с деформирующейся стенкой // Изв. АН СССР. Механика жидкости и газа. - 1968. - № 4. - С. 202-204.

13. Регирер С. А. Квазиодномерная теория перистальтических течений // Изв. АН СССР. Механика жидкости и газа. - 1984. - № 5. - С. 89-97.

14. Takagi D., Balmforth N. J. Peristaltic pumping of rigid objects in an elastic tube // J. Fluid Mech. - 2011. - Vol. 672. - P. 219-244. - DOI: 10.1017/S0022112010005926.

15. Yin F. C. P., Fung Y. C. Peristaltic waves in circular cylindrical tubes // Trans. ASME. J. Appl. Mech. - 1969. - Vol. 36. - P. 579-587.

16. Педли Т. Гидродинамика крупных кровеносных сосудов. - М. : Мир, 1983. - 400 с.

17. Медведев А. Е. Трехмерное движение вязкой несжимаемой жидкости в узкой трубке // Прикладная механика и техническая физика. - 2009. - Т. 50, № 4 (296). - С. 28-32.

18. Медведев А. Е. Нестационарное движение вязкой несжимаемой жидкости в трубке с деформирующейся стенкой // Прикладная механика и техническая физика. - 2013. - Т. 54, № 4 (320). - C. 45-54.

19. Тверье В. М., Гладышева О. С. Биомеханическая модель молочной железы // Master's Journal. - 2013. - № 2. - C. 240-252.

20. Гавриленко С. Л., Васин Р. А., Шилько С. В. Метод описания течения и определения реологических констант вязкопластических биоматериалов. Ч. 1 // Российский журнал биомеханики. - 2002. - Т. 6, № 3. - С. 92-99.

21. Метод описания течения и определения реологических констант вязкопластических биоматериалов. Ч. 2 \ С. В. Шилько, С. Л. Гавриленко, В. Ф. Хиженок, И. Н. Стакан, С. П. Саливончик // Российский журнал биомеханики. - 2003. - Т. 7, № 2. - С. 79-84. 
22. Goldstein S. Modern Developments in Fluid Mechanics. - Oxford : Oxford Univ. Press, 1938.

23. Neto C., Evans D., Bonaccurso E. Boundary slip in Newtonian liquids: a review of experimental studies // Reports on Progress in Physics. - 2005. - Vol. 39. - P. 2859-2897. DOI: $10.1088 / 0034-4885 / 68 / 12 / R 05$.

24. Переработка волокнообразующих полимеров. Основы реологии полимеров и течение полимеров в каналах / В. И. Янков, В. И. Боярченко, В. П. Перевадчук, И. О. Глот, Н. В. Шакиров. - М.-Ижевск : Изд-во «РХД», 2008. - 264 с.

25. Vinogradova O. I., Belyaev A. V. Wetting, roughness and flow boundary conditions // J. Phys.: Condens. Matter. - 2011. - Vol. 23. - P. 184104 (1-15).

26. Belyaev A. V., Vinogradova O. I. Effective slip in pressure-driven flow past super-hydrophobic stripes // J. Fluid Mech. - 2010. - Vol. 652. - P. 489-499. - DOI: 10.1017/S0022112010000741.

27. Борзенко Е. И., Дьякова О. А., Шрагер Г. Р. Исследование явления проскальзывания в случае течения вязкой жидкости в изогнутом канале // Вестник ТГУ. Механика. - 2014. № 2 (28). - C. 35-44.

28. Itoh S., Tanaka N., Tani A. The initial value problem for the Navier-Stokes equations with general slip boundary conditions in Holder spaces // J. Math, Fluid, Mech. - 2003. - Vol. 5. P. 275-301. - DOI: 10.1007/s00021-003-0074-6.

29. Beirao da Veiga H. Regularity for Stokes and generalized Stokes systems under nonhomogeneous slip-type boundary conditions // Advances in Differential Equations. - 2004. - Vol. 9, nos. 9-10. - P. 1079-1114.

30. Fugita H. Non-stationary Stokes flows under leak boundary conditions of friction type // J. Comput. Math. - 2001. - Vol. 19, № 1. - P. 1- 8 .

31. Гершуни Г. З., Жуховицкий Е. М. Конвективная устойчивость несжимаемой жидкости. - Москва : Наука, 1972. - 392 с.

32. Бурмашева Н. В., Просвиряков Е. Ю. Крупномасштабная слоистая стационарная конвекция вязкой несжимаемой жидкости под действием касательных напряжений на верхней границе. Исследование поля скоростей // Вестн. Самарск. гос. техн. унив. Сер. физ.-мат. науки. - 2017. - Т. 21, № 1. - C. 180-196. - DOI: 10.14498/vsgtu1527.

33. Privalova V. V., Prosviryakov E. Yu. Couette-Hiemenz Exact Solutions for the Steady Creeping Convective Flow of a Viscous Incompressible Fluid, with Allowance Made for Heat Recovery // J. Samara State Tech. Univ., Ser. Phys. Math. Sci. - 2018. - Vol. 22, no. 3. - P. 1-17. DOI: $10.14498 /$ vsgtu 1638 .

34. Privalova V. V., Prosviryakov E. Yu. Exact solutions for the convective creep CouetteHiemenz flow with the linear temperature distribution on the upper border // Diagnostics, Resource and Mechanics of materials and structures - 2018. - Iss. 2. - P. 92-109. - DOI: 10.17804/24109908.2018.2.092-109.

35. Aristov S.N., Prosviryakov E.Yu. Unsteady layered vortical fluid flows // Fluid Dynamics. 2016. - Vol. 51, no. 2. - P. 148-154. - DOI: 10.1134/S0040579516030027.

36. Navier C. L. M. H. M'emoire sur les Lois du Mouvement des Fluides // M'em. Acad. Sci. Inst. de France. - 1822. - Vol. 2, no. 6. - P. 389-440.

37. Navier C. L. M. H. Sur les lois du mouvement des fluids // M'em. Acad. R. Sci. inst. - 1827. Fr. 6. - P. 389-440. 